\title{
Clinical usefulness of bioimpedance analysis for assessing volume status in patients receiving maintenance dialysis
}

Jung Hwan Park, Young-Il Jo, and Jong-Ho Lee

Division of Nephrology, Department of Internal Medicine, Konkuk University School of Medicine, Seoul, Korea

Received: June 11, 2018 Accepted: June 15, 2018

\section{Correspondence to} Jung Hwan Park, M.D. Division of Nephrology, Department of Internal Medicine, Konkuk University Medical Center, 120-1 Neungdong-ro, Gwangjin-gu, Seoul 05030, Korea Tel: $+82-2-2030-7528$

Fax: +82-2-2030-7748

E-mail: pjh@kuh.ac.kr

This paper was contributed by Korean Society of Nephrology.
Chronic volume overload is associated with left ventricular hypertrophy and high cardiovascular mortality in patients undergoing dialysis. Therefore, estimating body fluid status is important in these patients. However, most dry-weight assessments are still performed clinically, while attempts have been made to measure the volume status and dry weight of patients undergoing dialysis using bioimpedance analysis (BIA). BIA uses the electrical properties of the human body to alternate current flow and measures resistance values to estimate body water content and composition. BIA is divided into single-frequency BIA, multi-frequency BIA, and bioimpedance spectroscopy (BIS) according to the number of frequencies used, and into whole-body and segmental BIA according to whether or not the whole body is divided into segments. Extracellular water (ECW), intracellular water, and total body water (TBW) contents can be measured with BIA. Dry weight can be estimated by measuring the volume overload of the patient through the ECW/TBW and ECW-to-body weight ratios. Other estimation methods include the normovolemia/hypervolemia slope method, a resistance-reactance (RXc) graph, overhydration measurements using a body composition monitor, and calf BIS. In this review, we will examine the principles of BIA, introduce various volume status measurement methods, and identify the optimal method for patients undergoing dialysis.

Keywords: Bioimpedance analysis; Dialysis; Dry weight; Body composition monitor

\section{INTRODUCTION}

The number of patients undergoing maintenance dialysis is increasing in Korea [1]. Volume status is an important prognostic factor of these patients. Overhydration $(\mathrm{OH})$ in patients undergoing dialysis is associated with the development of congestive heart failure and a higher frequency of mortality [2-4]. In contrast, dialysis-induced volume depletion and hypotension are common complications in patients undergoing hemodialysis (HD) and are independent risk factors for mor- tality $[5,6]$. Accurate assessment of volume status is required to manage volume, but this is a difficult task in patients undergoing dialysis. Clinical indices, such as blood pressure (BP), pulse rate, and edema, do not accurately reflect volume status. Some volume can remain in the body, and clinical edema may not be evident. Dry weight is defined as the weight at which a patient undergoing dialysis has neither edema nor hypertension without taking a BP-lowering medication, or the lowest weight that can be tolerated without developing symptoms of hypovolemia $[7,8]$. However, determining dry 
weight is a challenge for nephrologists because of the above-mentioned problems. Methods for determining dry weight have been suggested, including by reference to biochemical parameters, such as atrial or brain natriuretic peptide levels [9], inferior vena cava diameter on ultrasonography [10], and blood volume monitoring [11]. However, dry weight estimates obtained using these methods are not accurate. Dilution methods, regarded as reference methods for measuring body fluid volumes, are as follows: deuterium for total body water (TBW) [12], bromide for extracellular water (ECW) [13], and radioactive potassium isotope $\left({ }^{40} \mathrm{~K}\right)$ for intracellular water (ICW) [14]. Although these methods can be accurate, they are invasive, as they require blood samples, and are expensive due to the cost of the isotope and performance of mass spectrometry. Furthermore, they cannot be repeated at short intervals due to the retention of residual tracer, and they cannot be used to measure volume variations over a short period. Thus, bioimpedance analysis (BIA) is largely used instead, being a simple, noninvasive, and inexpensive method [15]. TBW, ICW, and ECW can be measured using BIA [16]. This method is precise and provides accurate estimates of TBW and ECW [17].

\section{PRINCIPLES OF BIOELECTRICAL IMPEDANCE}

BIA is based on the electrical properties of biological tissues [18]. When sending a weak alternating electric current into the body, electricity flows along highly conductive body tissues [19]. Current flows by two mechanisms: directly through the fluid and indirectly across cell membranes [20]. Low frequency current does not pass through cells; instead, current flows through the extracellular fluid [21]. However, the cell membrane capacitor charges and discharges the current at a high frequency. Thus, current flows through the cell membrane and tissue fluid (Fig. 1). The volume of water determines the width of the passage through which electricity flows, which is represented by impedance. Impedance $(Z)$ is a force that interferes with the flow of electric current and is determined using the vector sum of the electrical resistance $(R)$ and reactance $\left(X_{c}\right)$. The component of impedance corresponding to flow through fluid is the resistance, and the component cor-

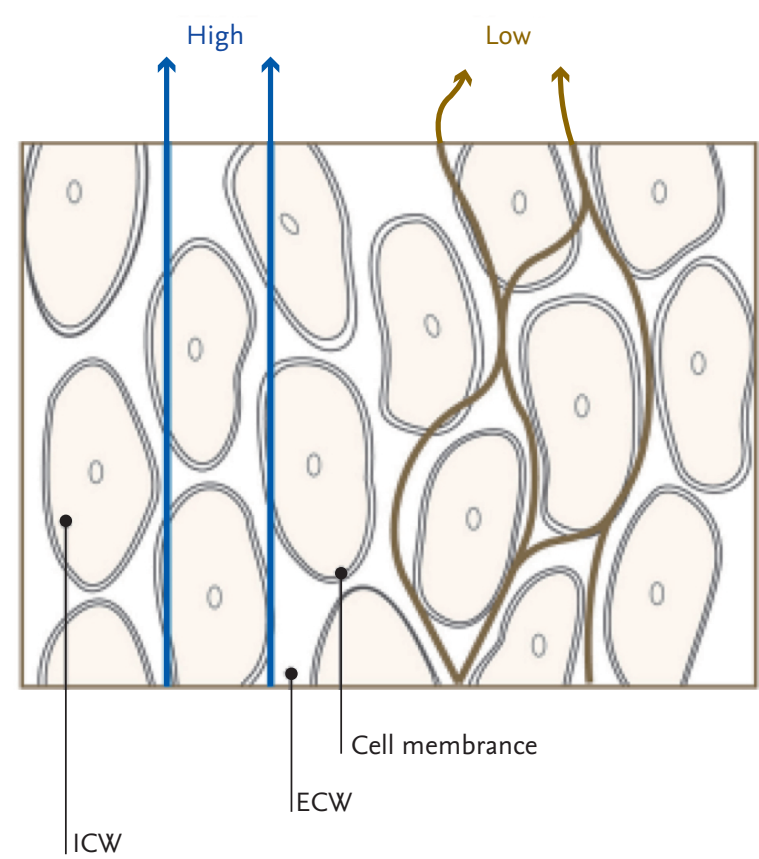

Figure 1. Current flows in tissue at low and high frequencies. Reproduced by courtesy of InBody [19]. ICW, intracellular water; ECW, extracellular water.

responding to flow across cell membranes is the reactance [22]. However, in the human body, the difference between the impedance and resistance values is only about 2 to $3 \Omega$ so the two words are often used interchangeably. The $R$ of the length of the conductive material is proportional to its length and inversely proportional to its cross-sectional area [15].

$$
\begin{aligned}
& R=\rho \frac{L}{A} \\
& R=\rho \frac{L \cdot L}{A \cdot L}=\rho \frac{L^{2}}{V} \\
& V=\rho \frac{L^{2}}{R}
\end{aligned}
$$

Length $(L)$ of the conductor is the length of the body, often replaced by the height. Resistivity $(\rho)$ is the resistance value per unit volume of body water. It is assumed that the resistivity value is constant because a highly constant amount of electrolyte is dissolved per unit volume in body water.

$$
\text { Body water volme }=C \times \frac{\text { Height }^{2}}{R}
$$




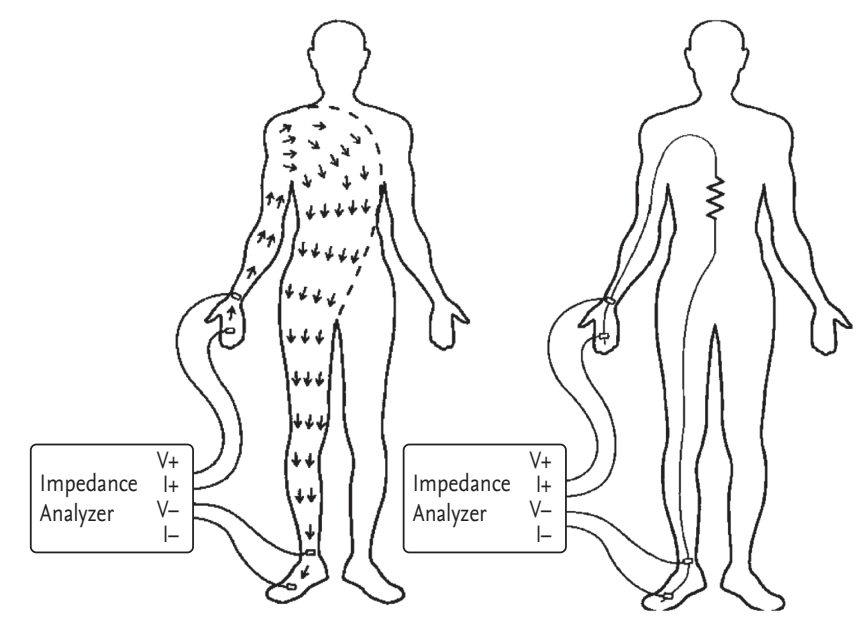

Figure 2. Measurement of body impedance. Reproduced by courtesy of InBody [19].

To measure impedance of the body, electrodes are attached to the right wrist, back of the hand, right ankle, and foot, as shown in Fig. 2, and connected to the impedance meter. The impedance meter supplies a current of $50 \mathrm{kHz}$ and $800 \mu \mathrm{A}$ between the electrodes of the back of the hand and the foot. Current flows through the right arm, torso, and right leg, where the impedance meter measures the voltage between the wrist and the ankle.

With the given current value and the measured voltage, the electric resistance value is obtained using Ohm's law.

$$
R=\frac{V}{i}
$$

After the height is measured, body water can be obtained by substituting the obtained resistance value of the body into (4).

\section{SINGLE-FREQUENCY BIA}

BIA information obtained at an electric current of 50 $\mathrm{KHz}$ is known as single-frequency BIA (SF-BIA). SF-BIA is the earliest and most frequently used methods to estimate the volume of body compartments [23]. SF-BIA predicts the volume of the TBW. However, strictly speaking, it does not measure TBW volume but rather the weighted sum of the ECW and ICW resistivities [15].
SF-BIA permits assessment of TBW and fat-free mass (FFM) for normal hydrated persons but is not appropriate under conditions of significantly altered hydration [24].

\section{MULTIPLE-FREQUENCY BIA}

Multiple-frequency BIA (MF-BIA) is a technology that precisely measures body water content, by measuring body water separately from ICW and ECW. Human impedance measured using high-frequency electrical signals reflects ICW and ECW, while low-frequency signals reflect only ECW. MF-BIA measures the distribution of water inside and outside the cell, by changing the frequency to in turn change the flow path of electricity. MF-BIA uses different frequencies ( 5 to $1,000 \mathrm{kHz}$ ) to evaluate FFM, TBW, ICW, and ECW. Hannan et al. [25] reported that TBW volume is more accurately estimated using MF-BIA than using the bioimpedance spectroscopy (BIS) method, which estimates ECW volume with equal accuracy. Patel et al. [26] reported that MFBIA was more accurate than SF-BIA for predicting ECW, whereas SF-BIA was more accurate for predicting TBW than MF-BIA in diseased subjects. MF-BIA shows less sensitivity in detecting fluid shifts between the ECW and the ICW in elderly patients [27].

\section{BIOIMPEDANCE SPECTROSCOPY}

BIS analyzes bioimpedance data obtained using a broadband of frequencies. The BIS method is based on determining resistance at zero and infinite frequencies, which are then used to predict ECW and TBW, respectively [23].

\section{WHOLE-BODY BIA}

Whole-body BIA regards the body as a cylinder having a certain length and cross-sectional area [18]. Its limitation is that it assumes that a body with arms, a trunk, and legs constitutes a single cylinder having uniform conductivity for any given cross-sectional area. The resistance to volume ratio differs between the trunk and 


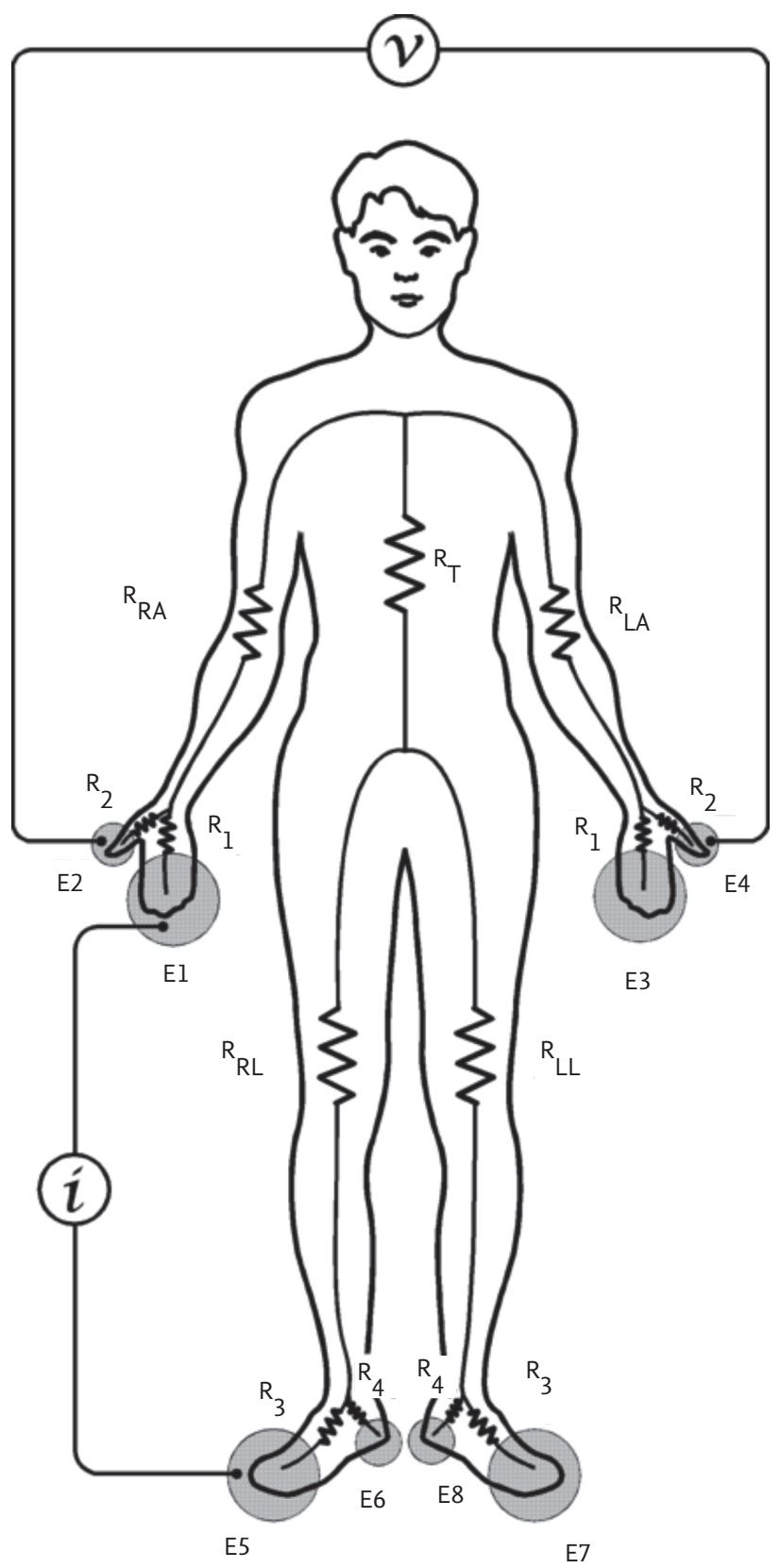

Figure 3. Measurement pathways of a segmental bioimpedance analysis device. Reproduced by courtesy of InBody [19].

the limbs. Limbs contribute $>90 \%$ of whole-body resistance despite having only $30 \%$ of the total volume, whereas the trunk, with a large cross-sectional area, contributes as little as $10 \%$ of whole-body resistance with $70 \%$ of the volume [28].

\section{SEGMENTAL BIA}

Segmental BIA has been developed to overcome the limitations of whole-body BIA. It regards the body as comprising five cylinders: two for the arms, two for the legs, and one for the trunk [18]. The device used to measure segmental BIA herein has up to eight electrodes (InBody, Seoul, Korea); two electrodes are in contact with the palm (E1, E3) and thumb (E2, E4) of each hand; and two are in contact with the anterior (E5, E7) and posterior aspects (E6, E8) of the sole of each foot (Fig. 3) [29]. The electrical resistance values of the right arm, left arm, trunk, right leg, and left leg are indicated by $R_{\mathrm{RA}}, R_{\mathrm{LA}}, R_{\mathrm{T}}, R_{\mathrm{RL}}$, and $R_{\mathrm{LL}}$, respectively. An alternating current of intensity $(I)$ is applied between E1 and E5. The voltage difference $(V)$ between $\mathrm{E}_{2}$ and $\mathrm{E}_{4}$ is divided by $I$ to obtain the resistance of the right arm. The current flows through $R_{1}-R_{R A}-R_{T}-R_{3}$, and the voltage is measured in a loop formed by $R_{2}-R_{\mathrm{RA}}-R_{\mathrm{LA}}-R_{2}$. The loop in which the current flows and the voltage measured overlap in $R_{R A}$ to measure resistance of the right arm [19]. Segmental BIA has been used to determine fluid shifts and distributions in certain conditions, such as ascites and kidney disease, as well as during surgery [15].

\section{ASSESSMENT OF FLUID OVERLOAD WITH BIA}

The normovolemic status of a subject can be estimated according to measurements of height, weight, and the body composition, as assessed by BIA. Fluid overload is calculated by subtracting the fluid volume according to the normovolemic status from that measured by BIA [30,31]. Several methods can be used to estimate fluid overload by BIA. Chamney et al. [30] used whole-body BIA to differentiate hypervolemia from normovolemia. A 70-kg healthy person has an ECW volume of 14 to 16 L [32]. They assumed that a linear relationship exists between ECW and body weight. The slope of this relationship has been denoted by the slope of normovolemia $\left(S_{\mathrm{NV}}\right)$ (Fig. 4). $S_{\mathrm{NV}}$ represents the average ECW volume for a given weight. All ingested fluid accumulates in the body and body weight increases in a patient undergoing dialysis. A second slope, the hypervolemic slope $\left(S_{\mathrm{HV}}\right)$, also develops under this hypervolemic condition. 


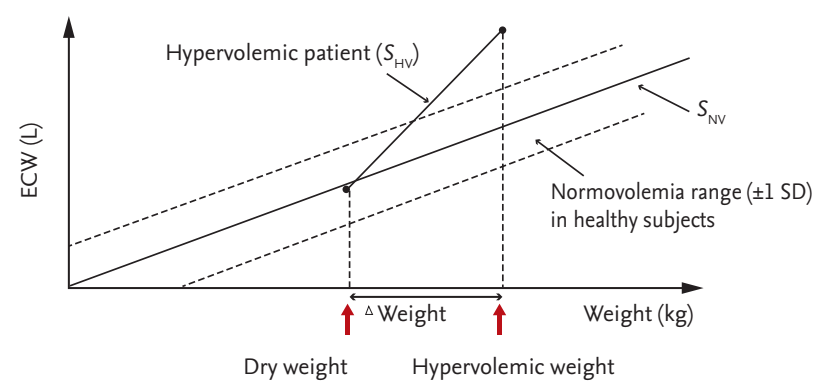

Figure 4. Relationship between normovolemic slope $\left(S_{\mathrm{NV}}\right)$ and hypervolemic slope $\left(S_{\mathrm{HV}}\right)$ with respect to body weight. Dry weight is indicated at the intersection of the $S_{\mathrm{NV}}$ and $\mathrm{S}_{\mathrm{HV}}$ slopes. Modified from Chamney et al, with permission of Elsevier Inc. [30].

Deducing the hydration status of a patient from the absolute body fluid volume alone is not appropriate because absolute water volume varies with height, weight, and body composition [33]. As an alternative, fluid compartment ratios, as indicators of hydration status, have been used including the ratio of ECW to TBW [34,35]. This ratio is easy to use, intuitive, and well validated as a predictor of survival [36]. However, the standard deviation of this ratio is so large that its use is impracticable [33]. Ratios that have been used by other researchers include ECW to body weight $[37,38]$, ECW to body height [38,39], and ECW to body surface area [40].

The resistance-reactance (RXc) graph method uses a direct impedance vector measurement (given by $R$ and $X c)$ at $50 \mathrm{kHz}$ with whole-body SF-BIA [41]. $R$ and $X c$ are plotted against each other and normalized by height. The $R / H$ and $X c / H(\Omega / m)$ ratios of a given patient are plotted on the RXc plane (Fig. 5). The vector length represents the size of the impedance. Clinical information on hydration is obtained by determining the location of the vector point for a normally hydrated healthy individual of the same sex, age, and race [42]. Vectors within the $75 \%$ tolerance ellipse indicate normal hydration status.

A body composition monitor (BCM, Fresenius Medical Care, Bad Homburg, Germany) is used to measure $\mathrm{OH}$. It uses two physiological models: in the volume model, the ECW and ICW are calculated using the resistance values measured with the BCM and Hanai model [31]; while in the body composition model, TBW is divided into three parts, i.e., $\mathrm{OH}$, lean tissue, and adipose tissue (Fig. 6) [43,44]. Absolute fluid overload (AFO; equivalent to $\mathrm{OH}$ ) is defined as the difference between

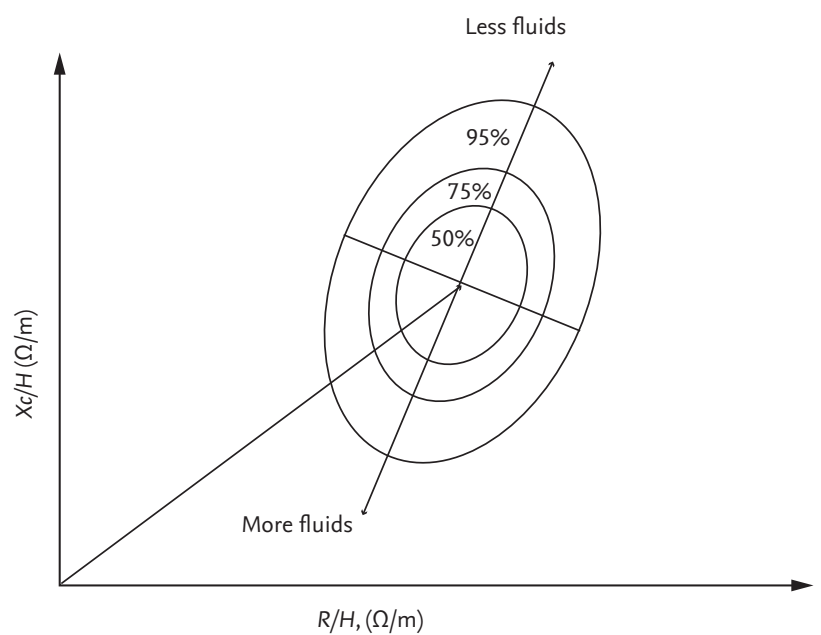

Figure 5. $R$ and $X c$, normalized for height, are plotted on the RXc graph with elliptical probability regions (50\%, 75\%, and $95 \%$ tolerance ellipses). Vector movement along the major axis of ellipses means changes in hydration status. Modified from Piccoli, with permission of Karger Publishers [41]. R, resistance; $X c$, reactance; $H$, height.

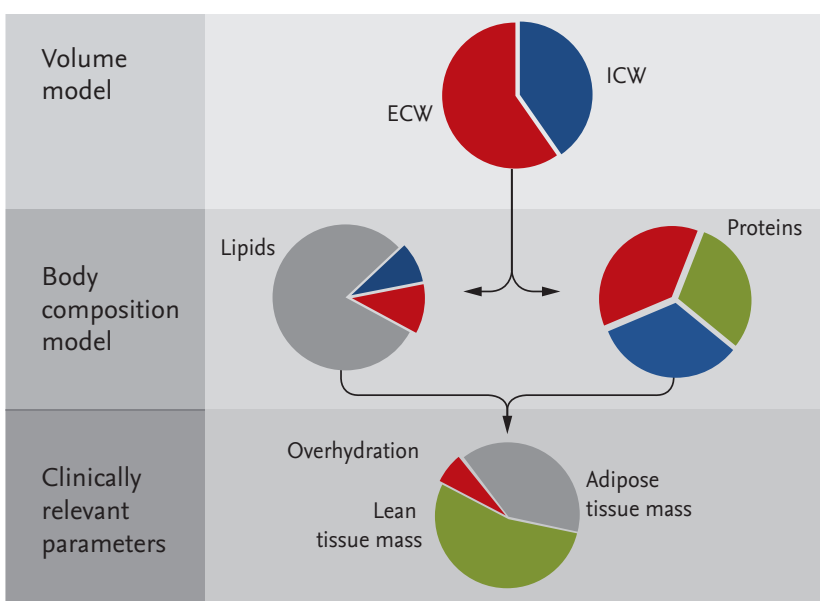

Figure 6. The volume model describes electrical conductance in a cell suspension enabling the total body water (TBW), extracellular water (ECW), and intracellular water (ICW) to be calculated. The body composition model is used for calculating the three relevant body compartments, namely overhydration, lean tissue, and adipose tissue, from ECW and TBW information. Reproduced by courtesy of Fresenius Medical Care [44].

the expected ECW under normal physiological conditions and the actual ECW, while relative fluid overload (RFO) is the AFO as a proportion of ECW (AFO/ECW) [45]. All calculations are performed automatically using BCM software. Normohydration is defined when the 
AFO is in the 1oth to 9oth percentile range; that is, -1.1 to $1.1 \mathrm{~L}$ relative to healthy age- and sex-matched individuals from the reference group. Volumes below and above this range are defined as underhydration and $\mathrm{OH}$, respectively.

\section{DRY WEIGHT ASSESSMENT BY BIA}

The normovolemia/hypervolemia slope method predicts dry weight from the BIS measurement and body weight (Fig. 4) [18,30]. All fluid is assumed to accumulate in the ECW. If the weight of a hypervolemic patient is reduced and $S_{\mathrm{HV}}$ intersects with $S_{\mathrm{NV}}$, the weight at which intersection occurs is the dry weight. In a study by Chamney et al. [30], the dialyzed weight of each patient typically decreased between 0.5 and $2 \mathrm{~kg}$ over subsequent dialyses and most patients achieve normovolemia in 2 to 6 weeks. A considerable improvement in BP control was achieved in that study, based on the mean arterial pressure, and there was an $86 \%$ reduction in the use of antihypertensive medication.

Chen et al. [46] used ECW\% (ECW as a percentage of weight) to determine the dry weight. ECW was measured by whole-body BIA. An ECW\% > 25\% in female patients and $>28 \%$ in male patients was considered excessive according to the rooth percentile of healthy subjects. The ECW\% of patients with a high BP was significantly higher than that of patients with a normal BP $(24.29 \% \pm 3.56 \%$ vs. $21.50 \% \pm 2.38 \%)$. All patients with an excessive ECW\% had high BPs, but not all patients with high BPs had an excessive ECW\%. No patient with a normal BP had an excessive ECW\%. Dry weight decreased in patients with a high BP and excessive ECW\%. ECW\% and BP decreased significantly after reducing dry weight. Dry weight increased in the symptomatic normotensive patients and symptoms improved in most of these patients (75\%).

The vector length in the RXc graph is regarded as a proxy for volume status and increases with ultrafiltration. Shorter predialysis bioimpedance vectors, indicating $\mathrm{OH}$, are associated with an increased mortality risk in patients undergoing HD [47]. Vectors situated within the $75 \%$ tolerance ellipse after ultrafiltration are assumed to indicate normal hydration in patients undergoing HD. However, vectors at the start and end of HD remain within the $75 \%$ tolerance ellipse in a relatively large proportion of patients [48].

Onofriescu et al. [45] used BCM to determine dry weight. They compared a bioimpedance group (volume controlled based on repeated BCM measurement in a 3-month period) with a clinical group over 2.5 years. RFO decreased significantly, from $9.52 \%$ to $7.46 \%$, in the bioimpedance group, whereas RFO did not decrease (10.30\% to $11.24 \%$ ) in the clinical group. Systolic BP decreased significantly, from 145.4 to $138.9 \mathrm{mmHg}$, in the bioimpedance group, whereas systolic BP decreased from 144.6 to $140.5 \mathrm{mmHg}$ in the clinical group; the decrease was not significant. In another study, these authors also reported that the cutoff RFO of $17.4 \%$ was associated with an increased risk of all-cause mortality in patients undergoing $\mathrm{HD}$ [49].

Machek et al. [50] conducted a study to determine the normohydration status of patients undergoing HD using BCM. The patients were categorized according to baseline hydration status, as follows: hyperhydrated group (RFO > 15\%); adverse event group (patients with more than two adverse events in the last 4 weeks); and all other patients. They targeted an RFO value of between $-6 \%$ and $6 \%$ postdialysis. Fluid overload was reduced by $2.0 \mathrm{~L}$ in the hyperhydrated group without increasing the number of intradialytic adverse events. Systolic BP was reduced by $25 \mathrm{mmHg}$, and a $35 \%$ reduction in antihypertensive medication use was achieved. Volume status was increased by $1.3 \mathrm{~L}$ in the adverse event group, and a $73 \%$ reduction in intradialytic adverse events was achieved without a significant increase in BP.

Moissl et al. [51] introduced the concept of weekly time-averaged fluid overload (TAFO), defined as the average of three predialysis fluid overload (FOpre) and postdialysis fluid overload (FOpost) values in a week, as follows: average weekly TAFO $=($ FOpre $1+$ FOpre $2+$ FOpre3 + FOpost 1 + FOpost $2+$ FOpost 3$) / 6$.

They used a target TAFO of $0.5 \mathrm{~L}$, according to the median value of more than 17,000 patients. They measured BCM on 3 days per week on which dialysis was performed. At the end of each week, the postdialysis target weights for the next week were calculated according to the following criteria:

(1) TAFO > 2.8 L: decrease postdialysis weight by $1 \mathrm{~kg} /$ week 


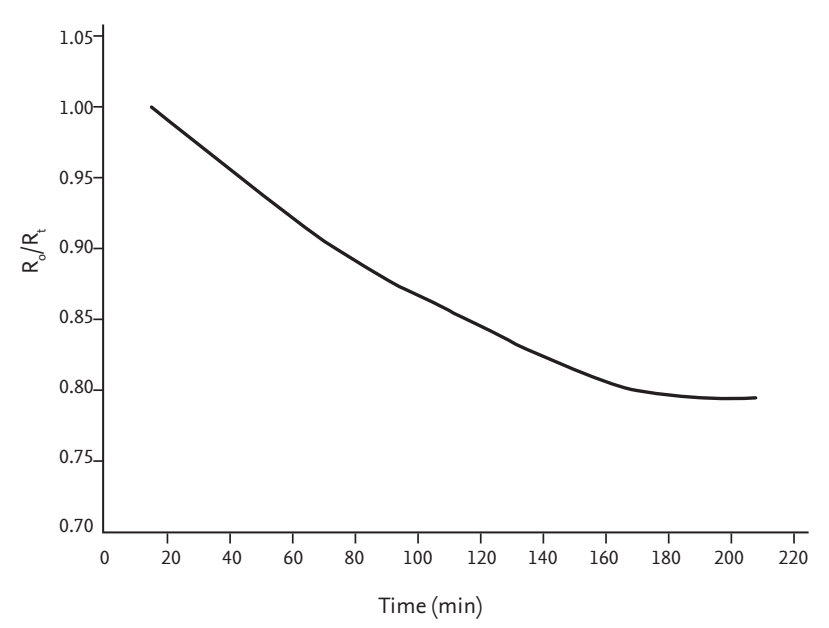

Figure 7. The calf-bioimpedance spectroscopy method that is used to estimate dry weight. The line represents the continuous value of the extracellular resistance ratio $\left(R_{\mathrm{o}} / R_{\mathrm{t}}\right)$. The flattening of the curve indicates that the excessive extracellular water in the patient's calf had been removed. Dry weight is defined as the flattening of the curve over a period of 20 minutes. Modified from $\mathrm{Zhu}$ et al. with permission of SAGE Publications [52].

(2) TAFO between 1.25 and 2.8 L: decrease postdialysis weight by $0.5 \mathrm{~kg} /$ week

(3) TAFO between -0.25 and $1.25 \mathrm{~L}$ (on target): maintain current dry weight

(4) TAFO <- - $0.25 \mathrm{~L}$ : increase postdialysis weight by 0.5 $\mathrm{kg} /$ week

The TAFO of all patients decreased from $0.9 \pm 1.6$ to $0.6 \pm 1.1 \mathrm{~L}$. TAFO decreased by $-1.20 \pm 1.32 \mathrm{~L}$ in the fluid overload group (baseline TAFO $>1.25 \mathrm{~L}$ above the target) and was unchanged in the normovolemic group (baseline TAFO between -0.25 and 1.25), but increased by 0.59 $\pm 0.76 \mathrm{~L}$ in the underhydrated group (baseline TAFO < $-0.25 \mathrm{~L}$ ). Every 1-L change in fluid overload was accompanied by a $9.9-\mathrm{mmHg} / \mathrm{L}$ change in the predialysis systolic BP. The number of intradialytic adverse events did not change significantly in any of the groups.

The calf-BIS method has been proposed as a method of measuring dry body weight. This is useful because the calf-ECW reflects whole-body ECW [52]. Calf-BIS is continuously measured during HD to identify the section wherein the $R_{0} / R_{t}$ curve becomes flat $\left(R_{0}\right.$ is the calf extracellular resistance at the HD start time, and $R_{\mathrm{t}}$ is the calf extracellular resistance at a specific time point) (Fig. 7). In this section, all excess ECW fluid was removed, and the weight was considered dry weight.

\section{BIA IN PATIENTS UNDERGOING PERITONEAL DIALYSIS}

Many patients undergoing peritoneal dialysis (PD) are in a state of $\mathrm{OH}[37,53]$. In patients undergoing PD, who lack the signs of dehydration observed in patients undergoing HD (such as hypotension or cramps during dialysis), it is more difficult to determine dry weight than in patients undergoing HD [33]. BIA is also a noninvasive, inexpensive, and simple way to measure body fluid in patients undergoing PD.

Lindley et al. [35] used BIS to estimate volume status in 31 patients undergoing PD and 199 healthy subjects. The ECW/TBW ratio of the patients undergoing PD was compared with that of healthy control subjects. The hydration score (difference between the measured ECW/TBW ratio and the mean ratio for age- and sexmatched controls divided by the standard deviation of the controls) was calculated. The mean hydration score was significantly higher (1.3 vs. 0.0) in the patients undergoing PD than in the control subjects. In total, $35 \%$ of the patients undergoing PD, and only $2.5 \%$ of the controls, had a hydration score $>2.0$. After a 3-month follow-up, a weight reduction of $3.6 \pm 2.3 \mathrm{~kg}$ had been achieved in seven patients through modality or prescription changes, with no adverse effects.

van de Kerkhof et al. [38] used MF-BIA to validate the predictive value of various ratios for diagnosing hypervolemia in patients undergoing dialysis. They divided the patients into the following groups: clinically normovolemic (mean 24- or 48-hour systolic BP $<133$ $\mathrm{mmHg}$ without antihypertensive agents), hypervolemic (mean systolic BP > $133 \mathrm{mmHg}$ with use of two or more antihypertensive agents), or undetermined. The 8oth percentile for normalized ECW in the clinically normovolemic patients was used as the reference value. The ECW/body height ratio had a higher sensitivity than the ECW/body weight ratio or ECW/TBW ratio for detecting hypervolemia in patients undergoing dialysis.

O'Lone et al. [54] showed that the OH/ECW ratio was an independent predictor of mortality in 529 patients undergoing PD, but the ECW/TBW ratio was not associated with increased mortality. Yoon et al. [55] reported that $\mathrm{OH}$ was associated with lower health-related quality of life in 481 patients undergoing PD.

A recent study suggested that the ratio of bioimpedance (RBI) might be a helpful parameter to adjust dry 
weight in patients undergoing PD [56]. RBI was calculated as follows: $\mathrm{RBI}=$ impedance at $50 \mathrm{kHz} /$ impedance at $500 \mathrm{kHz}$. RBI was measured from the right wrist to the right ankle by BCM. The patients were divided into hypervolemic and non-hypervolemic groups. The mean RBI of the non-hypervolemic patients was higher than that of the hypervolemic patients. They concluded that RBI could serve as a new index for estimating the volume status of patients undergoing PD.

\section{CONCLUSIONS}

Various BIA devices are increasingly being used in clinical practice, such as for estimating dry weight in patients undergoing dialysis. In patients undergoing $\mathrm{HD}$ or PD, volume overload commonly occurs, and identifying and reducing volume overload with BIA devices improves the prognosis by decreasing the BP and left ventricular hypertrophy [51,57-6o]. In addition, adverse events can be decreased in patients with a depleted volume by detecting their volume status and increasing the dry weight. However, the accuracy of $\mathrm{OH}$ measurement is the most important consideration.

The normovolemia/hypervolemia slope method is a useful method for determining dry weight, and reducing the variation in $S_{\mathrm{NV}}$ is key to its accuracy. Ratio values, such as ECW\%, are intuitive and easy to use, but both values can be abnormal because it is a ratio. The RXc graph has the advantage of yielding a relatively simple dry weight prediction without the need to measure volume status. However, as described earlier, it is sometimes difficult to determine dry weight because the vector can be within the $75 \%$ tolerance ellipse at the beginning and end of the dialysis. The calf-BIS method allows continuous monitoring during dialysis and provides relatively accurate data on hydration status, although measuring calf circumference is laborious. BCM methods are currently the most widely used and have been validated in various ways.

In conclusion, BIA is a useful method for controlling volume in patients undergoing dialysis, but more research is needed to establish a standard method.

\section{Conflict of interest}

No potential conflict of interest relevant to this article was reported.

\section{Acknowledgments}

This manuscript was written as part of Konkuk University's research support program for its faculty on sabbatical leave in 2010.

\section{REFERENCES}

1. Jin DC, Yun SR, Lee SW, Han SW, Kim W, Park J. Current characteristics of dialysis therapy in Korea: 2015 registry data focusing on elderly patients. Kidney Res Clin Pract 2016;35:204-211.

2. Parfrey PS, Harnett JD, Griffiths SM, Gault MH, Barre PE. Congestive heart failure in dialysis patients. Arch Intern Med 1988;148:1519-1525.

3. Wizemann V, Wabel P, Chamney P, et al. The mortality risk of overhydration in haemodialysis patients. Nephrol Dial Transplant 2009;24:1574-1579.

4. Kim YJ, Jeon HJ, Kim YH, et al. Overhydration measured by bioimpedance analysis and the survival of patients on maintenance hemodialysis: a single-center study. Kidney Res Clin Pract 2015;34:212-218.

5. Barth C, Boer W, Garzoni D, et al. Characteristics of hypotension-prone haemodialysis patients: is there a critical relative blood volume? Nephrol Dial Transplant 2003;18:1353-1360.

6. Shoji T, Tsubakihara Y, Fujii M, Imai E. Hemodialysis-associated hypotension as an independent risk factor for two-year mortality in hemodialysis patients. Kidney Int 2004;66:1212-1220.

7. Thomson GE, Waterhouse K, McDonald HP Jr, Friedman EA. Hemodialysis for chronic renal failure. Clinical observations. Arch Intern Med 1967;120:153-167.

8. Vasko R, Muller GA, Ratliff BB, Jung K, Gauczinski S, Koziolek MJ. Clinical judgment is the most important element in overhydration assessment of chronic hemodialysis patients. Clin Exp Nephrol 2013;17:563-568.

9. Lee SW, Song JH, Kim GA, Lim HJ, Kim MJ. Plasma brain natriuretic peptide concentration on assessment of hydration status in hemodialysis patient. Am J Kidney Dis 2003;41:1257-1266.

10. Ando Y, Yanagiba S, Asano Y. The inferior vena cava diameter as a marker of dry weight in chronic hemodialyzed patients. Artif Organs 1995;19:1237-1242. 
11. Steuer RR, Germain MJ, Leypoldt JK, Cheung AK. Enhanced fluid removal guided by blood volume monitoring during chronic hemodialysis. Artif Organs 1998;22:627-632.

12. Schloerb PR, Friis-hansen BJ, Edelman IS, Solomon AK, Moore FD. The measurement of total body water in the human subject by deuterium oxide dilution; with a consideration of the dynamics of deuterium distribution. J Clin Invest 1950;29:1296-1310.

13. Miller ME, Cosgriff JM, Forbes GB. Bromide space determination using anion-exchange chromatography for measurement of bromide. Am J Clin Nutr 1989;50:168-171.

14. Pierson RN Jr, Wang J, Colt EW, Neumann P. Body composition measurements in normal man: the potassium, sodium, sulfate and tritium spaces in 58 adults. J Chronic Dis 1982;35:419-428.

15. Kyle UG, Bosaeus I, De Lorenzo AD, et al. Bioelectrical impedance analysis: part I: review of principles and methods. Clin Nutr 2004;23:1226-1243.

16. Hannan WJ, Cowen SJ, Fearon KC, Plester CE, Falconer JS, Richardson RA. Evaluation of multi-frequency bio-impedance analysis for the assessment of extracellular and total body water in surgical patients. Clin Sci (Lond) 1994;86:479-485.

17. Sartorio A, Malavolti M, Agosti F, et al. Body water distribution in severe obesity and its assessment from eight-polar bioelectrical impedance analysis. Eur J Clin Nutr 2005;59:155-16o.

18. Kuhlmann MK, Zhu F, Seibert E, Levin NW. Bioimpedance, dry weight and blood pressure control: new methods and consequences. Curr Opin Nephrol Hypertens 2005;14:543-549.

19. Cha K. Principles of bioelectritical impedance analysis. Seoul (KR): InBody Co. Ltd., 1977:1-8.

20. Matthie J, Zarowitz B, De Lorenzo A, et al. Analytic assessment of the various bioimpedance methods used to estimate body water. J Appl Physiol (1985) 1998;84:1801-1816.

21. Buendia R, Seoane F, Lindecrantz K, et al. Estimation of body fluids with bioimpedance spectroscopy: state of the art methods and proposal of novel methods. Physiol Meas 2015;36:2171-2187.

22. Tattersall J. Bioimpedance analysis in dialysis: state of the art and what we can expect. Blood Purif 2009;27:70-74.

23. Khalil SF, Mohktar MS, Ibrahim F. The theory and fundamentals of bioimpedance analysis in clinical status monitoring and diagnosis of diseases. Sensors (Basel)
2014;14:10895-10928.

24. Lukaski HC, Bolonchuk WW, Hall CB, Siders WA. Validation of tetrapolar bioelectrical impedance method to assess human body composition. J Appl Physiol (1985) 1986;60:1327-1332.

25. Hannan WJ, Cowen SJ, Plester CE, Fearon KC, deBeau A. Comparison of bio-impedance spectroscopy and multi-frequency bio-impedance analysis for the assessment of extracellular and total body water in surgical patients. Clin Sci (Lond) 1995;89:651-658.

26. Patel RV, Peterson EL, Silverman N, Zarowitz BJ. Estimation of total body and extracellular water in post-coronary artery bypass graft surgical patients using single and multiple frequency bioimpedance. Crit Care Med 1996;24:1824-1828.

27. Olde Rikkert MG, Deurenberg P, Jansen RW, van't Hof MA, Hoefnagels WH. Validation of multi-frequency bioelectrical impedance analysis in detecting changes in fluid balance of geriatric patients. J Am Geriatr Soc 1997;45:1345-1351.

28. Abbas SR, Zhu F, Levin NW. Bioimpedance can solve problems of fluid overload. J Ren Nutr 2015;25:234-237.

29. Bedogni G, Malavolti M, Severi S, et al. Accuracy of an eight-point tactile-electrode impedance method in the assessment of total body water. Eur J Clin Nutr 2002;56:1143-1148.

30. Chamney PW, Kramer M, Rode C, Kleinekofort W, Wizemann V. A new technique for establishing dry weight in hemodialysis patients via whole body bioimpedance. Kidney Int 2002;61:2250-2258.

31. Moissl UM, Wabel P, Chamney PW, et al. Body fluid volume determination via body composition spectroscopy in health and disease. Physiol Meas 2006;27:921-933.

32. Chertow GM, Lowrie EG, Wilmore DW, et al. Nutritional assessment with bioelectrical impedance analysis in maintenance hemodialysis patients. J Am Soc Nephrol 1995;6:75-81.

33. Zhu F, Wystrychowski G, Kitzler T, Thijssen S, Kotanko P, Levin NW. Application of bioimpedance techniques to peritoneal dialysis. Contrib Nephrol 2006;150:119-128.

34. Asghar RB, Green S, Engel B, Davies SJ. Relationship of demographic, dietary, and clinical factors to the hydration status of patients on peritoneal dialysis. Perit Dial Int 2004;24:231-239.

35. Lindley E, Devine Y, Hall L, et al. A ward-based procedure for assessment of fluid status in peritoneal dialysis pa- 
tients using bioimpedance spectroscopy. Perit Dial Int 2005;25 Suppl 3:S46-S48.

36. Davies SJ, Davenport A. The role of bioimpedance and biomarkers in helping to aid clinical decision-making of volume assessments in dialysis patients. Kidney Int 2014;86:489-496.

37. Konings CJ, Kooman JP, Schonck M, et al. Fluid status, blood pressure, and cardiovascular abnormalities in patients on peritoneal dialysis. Perit Dial Int 2002;22:477487.

38. van de Kerkhof J, Hermans M, Beerenhout C, et al. Reference values for multifrequency bioimpedance analysis in dialysis patients. Blood Purif 2004;22:301-306.

39. Wang X, Axelsson J, Lindholm B, Wang T. Volume status and blood pressure in continuous ambulatory peritoneal dialysis patients. Blood Purif 2005;23:373-378.

40. Plum J, Schoenicke G, Kleophas W, et al. Comparison of body fluid distribution between chronic haemodialysis and peritoneal dialysis patients as assessed by biophysical and biochemical methods. Nephrol Dial Transplant 2001;16:2378-2385.

41. Piccoli A. Whole body: single frequency bioimpedance. Contrib Nephrol 2005;149:150-161.

42. Piccoli A, Rossi B, Pillon L, Bucciante G. A new method for monitoring body fluid variation by bioimpedance analysis: the RXc graph. Kidney Int 1994;46:534-539.

43. Chamney PW, Wabel P, Moissl UM, et al. A whole-body model to distinguish excess fluid from the hydration of major body tissues. Am J Clin Nutr 2007;85:80-89.

44. Fresenius Medical Care. BCM standard. Bad Homburg (DE): Fresenius Medical Care, 2014.

45. Onofriescu M, Hogas S, Voroneanu L, et al. Bioimpedance-guided fluid management in maintenance hemodialysis: a pilot randomized controlled trial. Am J Kidney Dis 2014;64:111-118.

46. Chen YC, Chen HH, Yeh JC, Chen SY. Adjusting dry weight by extracellular volume and body composition in hemodialysis patients. Nephron 2002;92:91-96.

47. Pillon L, Piccoli A, Lowrie EG, Lazarus JM, Chertow GM. Vector length as a proxy for the adequacy of ultrafiltration in hemodialysis. Kidney Int 2004;66:1266-1271.

48. Piccoli A. Identification of operational clues to dry weight prescription in hemodialysis using bioimpedance vector analysis. The Italian Hemodialysis-Bioelectrical Impedance Analysis (HD-BIA) Study Group. Kidney Int
1998:53:1036-1043.

49. Onofriescu M, Siriopol D, Voroneanu L, et al. Overhydration, cardiac function and survival in hemodialysis patients. PLoS One 2015;10:e0135691.

50. Machek P, Jirka T, Moissl U, Chamney P, Wabel P. Guided optimization of fluid status in haemodialysis patients. Nephrol Dial Transplant 2010;25:538-544.

51. Moissl U, Arias-Guillen M, Wabel P, et al. Bioimpedance-guided fluid management in hemodialysis patients. Clin J Am Soc Nephrol 2013;8:1575-1582.

52. Zhu F, Kuhlmann MK, Sarkar S, et al. Adjustment of dry weight in hemodialysis patients using intradialytic continuous multifrequency bioimpedance of the calf. Int J Artif Organs 2004;27:104-109.

53. Oe B, De Fijter CW, Geers TB, Vos PF, de Vries PM. Hemodialysis (HD) versus peritoneal dialysis (PD): latent overhydration in PD patients? Int J Artif Organs 2002;25:838-843.

54. O'Lone EL, Visser A, Finney H, Fan SL. Clinical significance of multi-frequency bioimpedance spectroscopy in peritoneal dialysis patients: independent predictor of patient survival. Nephrol Dial Transplant 2014;29:1430-1437.

55. Yoon HE, Kwon YJ, Song HC, et al. Overhydration negatively affects quality of life in peritoneal dialysis patients: evidence from a prospective observational study. Int J Med Sci 2016;13:686-695.

56. Jang M, Kim WH, Lee JH, et al. Numerical expression of volume status using the bioimpedance ratio in continuous ambulatory peritoneal dialysis patients: a pilot study. Kidney Res Clin Pract 2017;36:290-295.

57. Foley RN, Parfrey PS, Morgan J, et al. Effect of hemoglobin levels in hemodialysis patients with asymptomatic cardiomyopathy. Kidney Int 2000;58:1325-1335.

58. Hur E, Usta M, Toz H, et al. Effect of fluid management guided by bioimpedance spectroscopy on cardiovascular parameters in hemodialysis patients: a randomized controlled trial. Am J Kidney Dis 2013;61:957-965.

59. Middleton RJ, Parfrey PS, Foley RN. Left ventricular hypertrophy in the renal patient. J Am Soc Nephrol 2001; 12:1079-1084.

6o. Chazot C, Wabel P, Chamney P, Moissl U, Wieskotten S, Wizemann V. Importance of normohydration for the long-term survival of haemodialysis patients. Nephrol Dial Transplant 2012;27:2404-2410. 